\title{
Counting subgroups of finite nonmetacyclic 2-groups having no elementary abelian subgroup of order 8
}

\author{
EniOluwafe, Michael \\ (Department of Mathematics/Faculty of Science/University of Ibadan, Nigeria)
}

Abstract:The aim of this note is to give an explicit formula for the number of subgroups of finite nonmetacyclic 2-groups having no elementary abelian subgroup of order 8.

Keywords:Centralproducts, cyclic subgroups, dihedral groups, finite nonmetacyclic 2-groups, number of subgroups.

\section{Introduction}

Counting subgroups of finite groups solves one of the most important problems of combinatorial finite group theory. For example, in [1] are determined an explicit expression for the number of subgroups of finite nonabelianp-groups having a cyclic subgroup.

Recall that the problem was completely solved in the abelian case, by establishing an explicit expression of the number of subgroups of a finite abelian group [2]. Unfortunately, in the nonabeliannonmetacyclic casea such expression can be given only for certain finite groups [3]. In this note we prove a counting theorem for a class of finite nonmetacyclic 2-groups having no elementary abelian subgroup of order 8.

A group $\mathrm{G}$ is said to be metacyclic if it contains a normal cyclic subgroup $\mathrm{C}$ such that $\mathrm{G} / \mathrm{C}$ is cyclic, otherwise it is said to be nonmetacyclic. Let $\mathrm{A}$ and $\mathrm{B}$ be groups, a central product of groups $\mathrm{A}$ and $\mathrm{B}$ is denoted by $A * B$, that is, $A * B=A B$ with $[A, B]=\{1\}$, where $[A, B]$ is a commutator subgroup generated by groups $A$ and $\mathrm{B}$.

For basic definitions and results on groups we refer the reader to [4], [5] and [6]. More precisely, we prove the following result in the next section.

Theorem 2.1. Let $\mathrm{G}=\mathrm{D} * \mathrm{Z}$, where $*$ is a central product, $\mathrm{D} \cong \mathrm{D}_{2^{\mathrm{n}}}$, a dihedral group of order $2^{\mathrm{n}}, \mathrm{n} \geq$ $3, \mathrm{Z} \cong \mathrm{C}_{4}$, a cyclic group of order 4 and $\mathrm{D} \cap \mathrm{Z}=\mathrm{Z}(\mathrm{D}), \mathrm{Z}(\mathrm{D})$ is the center of $\mathrm{D}$. Then the number of subgroups of the group $\mathrm{G}$ is given by the following equality:

$$
|L(G)|= \begin{cases}23 & ; \text { ifn }=3 \\ 3\left(2+n+\sum_{k=2}^{n-2} 2^{n-k}\right)+2^{n} & ; \text { ifn } \geq 4\end{cases}
$$

where $L(G)$, the set consisting of all subgroups of $G$ forms a complete lattice with respect to set inclusion, called the subgroup lattice of $\mathrm{G}$.

\section{Proof of Theorem 2.1}

Proof. Let $\mathrm{D} \cong \mathrm{D}_{2^{\mathrm{n}}}=\left\langle\mathrm{x}, \mathrm{y} \mid \mathrm{x}^{2^{\mathrm{n}-1}}=\mathrm{y}^{2}=1, \mathrm{yxy}^{-1}=\mathrm{x}^{2^{\mathrm{n}-1}-1}\right\rangle, \mathrm{n} \geq 3$, a dihedral group of order $2^{\mathrm{n}}, \mathrm{Z} \cong \mathrm{C}_{4}=\langle\mathrm{a}\rangle$, a cyclic group of order 4 and $\mathrm{D} \cap \mathrm{Z}=\mathrm{Z}(\mathrm{D})$. Then $\mathrm{G}=\mathrm{D} * \mathrm{Z}:=\frac{\mathrm{D} \times \mathrm{Z}}{\mathrm{H}}$, where $\mathrm{H}=$ $\left\langle\left(\mathrm{x}^{2^{\mathrm{n}-2}}, \mathrm{a}^{2}\right)\right\rangle, \mathrm{n} \geq 3$. That is:

$$
\mathrm{G}:=\langle(\mathrm{x}, 1) \mathrm{H},(\mathrm{y}, 1) \mathrm{H},(1, \mathrm{a}) \mathrm{H}\rangle
$$

such that:

$$
\left(\mathrm{x}^{2^{\mathrm{n}-1}}, 1\right) \mathrm{H}=\left(\mathrm{y}^{2}, 1\right) \mathrm{H}=\left(1, \mathrm{a}^{4}\right) \mathrm{H}=\mathrm{H}, \quad\left(\mathrm{yxy}^{-1}, 1\right) \mathrm{H}=\left(\mathrm{x}^{2^{\mathrm{n}-1}-1}, 1\right) \mathrm{H}
$$

An important property of this group is that its characteristic subgroup defined by: $\mho_{n-2}(G):=$ $\left\langle\left(\mathrm{x}^{\mathrm{q}}, 1\right) \mathrm{H}\right\rangle$, where $\mathrm{q}=2^{\mathrm{n}-2}$, for all $\mathrm{n} \geq 3$, is of order 2 . Also, for $\mathrm{n} \geq 3$, we obtain an epimorphism $\delta: \mathrm{G} \rightarrow$ $\mathrm{D}_{2^{\mathrm{n}-1} \times} \mathrm{C}_{2}$ defined by:

$\delta(k H):=(k H)\left\langle\left(x^{2^{n-2}}, 1\right) H\right\rangle, n \geq 3$, where $k H \in G, k \in D \times \operatorname{Zand}(k H)\left\langle\left(x^{2^{n-2}}, 1\right) H\right\rangle \in D_{2^{n-1} \times} C_{2}, n \geq 3$. Clearly, the kernel of $\delta$ is

$\mho_{n-2}(G):=\left\langle\left(x^{2^{n-2}}, 1\right) H\right\rangle$ and by the first isomorphism theorem for groups, we obtain that: 


$$
\frac{\mathrm{G}}{\mho_{\mathrm{n}-2}(\mathrm{G})} \cong \mathrm{D}_{2^{\mathrm{n}-1} \times} \mathrm{C}_{2} \text { for alln } \geq 3
$$

Being isomorphic, the groups $\frac{G}{\mho_{n-2}(G)}$ and $2_{2^{n-1} \times} C_{2}$ have isomorphic lattices of subgroups.

Moreover, since the number of subgroups $G$ which not contain $\mho_{n-2}(G)$ are the trivial subgroup as well as all minimal subgroups of $G$ excepting $\mho_{n-2}(G)$ and since the distinct subgroups generated by the join of any two distinct such subgroups includes $\mho_{n-2}(G)$.

One obtains:

$$
|\mathrm{L}(\mathrm{G})|=\left|\mathrm{L}\left(\frac{\mathrm{G}}{\mho_{\mathrm{n}-2}(\mathrm{G})}\right)\right|+2^{\mathrm{n}-1}+3, \text { for alln } \geq 3
$$

Thus, we need to determine the number of subgroups of $\mathrm{D}_{2^{\mathrm{n}-1} \times} \mathrm{C}_{2}$ using the following auxiliary result established in [3].

Lemma 2.2: For all $n \geq 3$, the number of all subgroups of order $2^{n}$ in the finite 2-group $D_{2^{n-1} \times} C_{2}$ is:

$$
\begin{cases}16 & ; \text { ifn }=3 \\ 2^{n-1}+3\left(n+1+\sum_{i=1}^{n-2} 2^{n-i}\right) & ; \text { ifn } \geq 4\end{cases}
$$

Hence, the relations (1), (2) and (3) give the explicit expression of

$$
|L(G)|= \begin{cases}23 & ; \text { ifn }=3 \\ 2^{n}+3\left(2+n+\sum_{k=2}^{n-2} 2^{n-k}\right) & ; \text { ifn } \geq 4\end{cases}
$$

\section{Conclusion}

In this short note we had worked on minimal subgroups and used a previous result (Lemma 2.2) to obtain a counting theorem for a class of finite nonmetacyclic 2-groups having no elementary abelian subgroup of order 8 . It is desirable to consider arbitrary nonabeliannonmetacyclic 2 -groups.

\section{References}

[1]. M. Tarnauceanu, Counting subgroups for a class of finite nonabelian p-groups. AnaleleUniversitaatii de Vest, TimisoaraSeriaMatematica - Informatica XLVI, 1, (2008), 147-152.

[2]. G. Bhowmik, Evaluation of the divisor function of matrices, ActaArithmetica 74(1996), $155-159$

[3]. M. EniOluwafe, Counting subgroups of nonmetacyclic groups of type $D_{2^{n-1} \times} C_{2}, n \geq 3$, submitted.

[4]. B. Huppert, Endlichegruppen I, II (Springer-Verlag, Berlin, 1967).

[5]. M. Suzuki, Group theory I, II (Springer-Verlag, Berlin, 1982, 1986)

[6]. H. Zassenhaus, Theory of groups (Chelsea, New York, 1949). 